\title{
Sequent Calculus, Derivability, Provability. Gödel's Completeness Theorem ${ }^{1}$
}

\author{
Marco B. Caminati ${ }^{2}$ \\ Mathematics Department "G.Castelnuovo" \\ Sapienza University of Rome \\ Piazzale Aldo Moro 5, 00185 Roma, Italy
}

\begin{abstract}
Summary. Fifth of a series of articles laying down the bases for classical first order model theory. This paper presents multiple themes: first it introduces sequents, rules and sets of rules for a first order language $\mathrm{L}$ as L-dependent types. Then defines derivability and provability according to a set of rules, and gives several technical lemmas binding all those concepts. Following that, it introduces a fixed set $\mathrm{D}$ of derivation rules, and proceeds to convert them to Mizar functorial cluster registrations to give the user a slick interface to apply them.

The remaining goals summon all the definitions and results introduced in this series of articles. First: D is shown to be correct and having the requisites to deliver a sensible definition of Henkin model (see [18]). Second: as a particular application of all the machinery built thus far, the satisfiability and Gödel completeness theorems are shown when restricting to countable languages. The techniques used to attain this are inspired from [18], then heavily modified with the twofold goal of embedding them into the more flexible framework of a variable ruleset here introduced, and of proving completeness of a set of rules more sparing than the one there used; in particular the simpler ruleset allowed to avoid the definition and tractation of free occurence of a literal, a fact which, along with shortening proofs, is remarkable in its own right. A preparatory account of some of the ideas used in the proofs given here can be found in [15].
\end{abstract}

MML identifier: FOMODEL4, version: $\underline{7.11 .07 \quad 4.160 .1126}$

The notation and terminology used here have been introduced in the following papers: [1], [3], [23], [22], [4], [6], [17], [11], [12], [13], [14], [7], [8], [5], [19], [16], [24], [2], [21], [9], [26], [28], [27], [20], [25], and [10].

\footnotetext{
${ }^{1}$ The author wrote this paper as part of his $\mathrm{PhD}$ thesis research and would like to thank Marco Pedicini for his encouragement and support.

${ }^{2}$ The author is the winner of the Mizar Prize for Young Researchers in 2011 for this article. 


\section{Formalization of the Notion of Derivability and Provability. Henkin's TheOREM for Arbitrary Languages}

For simplicity, we adopt the following convention: $k, m, n$ denote natural numbers, $m_{1}$ denotes an element of $\mathbb{N}, U$ denotes a non empty set, $A, B, X$, $Y, Z, x, y, z$ denote sets, $S$ denotes a language, $s$ denotes an element of $S, f$, $g$ denote functions, $p_{1}, p_{2}, p_{3}, p_{4}$ denote w.f.f. strings of $S, P_{1}, P_{2}, P_{3}$ denote subsets of AllFormulas Of $S, t, t_{1}, t_{2}$ denote termal strings of $S, a$ denotes an of-atomic-formula element of $S, l, l_{1}, l_{2}$ denote literal elements of $S, p$ denotes a finite sequence, and $m_{2}$ denotes a non zero natural number.

Let $S$ be a language. The functor $S$-sequents is defined as follows:

(Def. 1) $S$-sequents $=\left\{\left\langle p_{5}, c_{1}\right\rangle ; p_{5}\right.$ ranges over subsets of AllFormulasOf $S, c_{1}$ ranges over w.f.f. strings of $S: p_{5}$ is finite $\}$.

Let $S$ be a language. Note that $S$-sequents is non empty.

Let us consider $S$. Observe that $S$-sequents is relation-like.

Let $S$ be a language and let $x$ be a set. We say that $x$ is $S$-sequent-like if and only if:

(Def. 2) $\quad x \in S$-sequents .

Let us consider $S, X$. We say that $X$ is $S$-sequents-like if and only if:

(Def. 3) $X \subseteq S$-sequents .

Let us consider $S$. One can check that every subset of $S$-sequents is $S$ sequents-like and every element of $S$-sequents is $S$-sequent-like.

Let $S$ be a language. One can verify that there exists an element of $S$-sequents which is $S$-sequent-like and there exists a subset of $S$-sequents which is $S$-sequents-like.

Let us consider $S$. One can check that there exists a set which is $S$-sequentlike and there exists a set which is $S$-sequents-like.

Let $S$ be a language. A rule of $S$ is an element of $\left(2^{S \text {-sequents }}\right)^{2^{S-\text { sequents }}}$.

Let $S$ be a language. A rule set of $S$ is a subset of $\left(2^{S \text {-sequents }}\right)^{2^{S-\text { sequents }}}$.

For simplicity, we adopt the following rules: $D, D_{1}, D_{2}, D_{3}$ denote rule sets of $S, R$ denotes a rule of $S, S_{1}, S_{2}, S_{3}$ denote subsets of $S$-sequents, $s_{1}, s_{2}, s_{3}$ denote elements of $S$-sequents, $S_{4}, S_{5}$ denote $S$-sequents-like sets, and $S_{6}, S_{7}$ denote $S$-sequent-like sets.

Let us consider $A, B$ and let $X$ be a subset of $B^{A}$. One can check that $\bigcup X$ is relation-like.

Let $S$ be a language and let $D$ be a rule set of $S$. One can check that $\cup D$ is relation-like.

Let us consider $S, D$. The functor OneStep $D$ yielding a rule of $S$ is defined as follows:

(Def. 4) For every element $S_{8}$ of $2^{S \text {-sequents }}$ holds $\quad($ OneStep $D)\left(S_{8}\right)=$ $\bigcup\left((\bigcup D)^{\circ}\left\{S_{8}\right\}\right)$. 
Let us consider $S, D, m$. The functor $(m, D)$-derivables yields a rule of $S$ and is defined by:

(Def. 5) $(m, D)$-derivables $=(\text { OneStep } D)^{m}$.

Let $S$ be a language, let $D$ be a rule set of $S$, and let $S_{9}, S_{10}$ be sets. We say that $S_{10}$ is $\left(S_{9}, D\right)$-derivable if and only if:

(Def. 6) $\quad S_{10} \subseteq \bigcup\left(\left((\text { OneStep } D)^{*}\right)^{\circ}\left\{S_{9}\right\}\right)$.

Let us consider $m, S, D$ and let $S_{1}, s_{1}$ be sets. We say that $s_{1}$ is $\left(m, S_{1}, D\right)$ derivable if and only if:

(Def. 7) $s_{1} \in(m, D)$-derivables $\left(S_{1}\right)$.

Let us consider $S, D$. The functor $D$-iterators yielding a family of subsets of $2^{S \text {-sequents }} \times 2^{S \text {-sequents }}$ is defined as follows:

(Def. 8) $D$-iterators $=\left\{(\text { OneStep } D)^{m_{1}}\right\}$.

Let us consider $S, R$. We say that $R$ is isotone if and only if:

(Def. 9) If $S_{2} \subseteq S_{3}$, then $R\left(S_{2}\right) \subseteq R\left(S_{3}\right)$.

Let us consider $S$. Observe that there exists a rule of $S$ which is isotone.

Let us consider $S, D$. We say that $D$ is isotone if and only if:

(Def. 10) For all $S_{2}, S_{3}, f$ such that $S_{2} \subseteq S_{3}$ and $f \in D$ there exists $g$ such that $g \in D$ and $f\left(S_{2}\right) \subseteq g\left(S_{3}\right)$.

Let us consider $S$ and let $M$ be an isotone rule of $S$. One can verify that $\{M\}$ is isotone.

Let us consider $S$. One can verify that there exists a rule set of $S$ which is isotone.

In the sequel $K, K_{1}$ are isotone rule sets of $S$.

Let $S$ be a language, let $D$ be a rule set of $S$, and let $S_{1}$ be a set. We say that $S_{1}$ is $D$-derivable if and only if:

(Def. 11) $S_{1}$ is $(\emptyset, D)$-derivable.

Let us consider $S, D$. One can verify that every set which is $D$-derivable is also $(\emptyset, D)$-derivable and every set which is $(\emptyset, D)$-derivable is also $D$-derivable.

Let us consider $S, D$ and let $S_{1}$ be an empty set. One can verify that every set which is $\left(S_{1}, D\right)$-derivable is also $D$-derivable.

Let us consider $S, D, X$ and let $p_{2}$ be a set. We say that $p_{2}$ is $(X, D)$-provable if and only if:

(Def. 12) $\quad\left\{\left\langle X, p_{2}\right\rangle\right\}$ is $D$-derivable or there exists a set $s_{1}$ such that $\left(s_{1}\right)_{1} \subseteq X$ and $\left(s_{1}\right)_{2}=p_{2}$ and $\left\{s_{1}\right\}$ is $D$-derivable.

Let us consider $S, D, X, x$. Let us observe that $x$ is $(X, D)$-provable if and only if:

(Def. 13) There exists a set $s_{1}$ such that $\left(s_{1}\right)_{1} \subseteq X$ and $\left(s_{1}\right)_{2}=x$ and $\left\{s_{1}\right\}$ is $D$-derivable.

Let us consider $S, D, R$. We say that $R$ is $D$-macro if and only if: 
(Def. 14) For every subset $S_{8}$ of $S$-sequents holds $R\left(S_{8}\right)$ is $\left(S_{8}, D\right)$-derivable.

Let us consider $S, D$ and let $P_{1}$ be a set. The functor $\left(P_{1}, D\right)$-termEq is defined as follows:

(Def. 15) $\quad\left(P_{1}, D\right)$-termEq $=\left\{\left\langle t_{1}, t_{2}\right\rangle ; t_{1}\right.$ ranges over termal strings of $S, t_{2}$ ranges over termal strings of $S$ : 〈TheEqSymbOf $S\rangle\urcorner t_{1} t_{2}$ is $\left(P_{1}, D\right)$-provable\}.

Let us consider $S, D$ and let $P_{1}$ be a set. We say that $P_{1}$ is $D$-expanded if and only if:

(Def. 16) If $x$ is $\left(P_{1}, D\right)$-provable, then $\{x\} \subseteq P_{1}$.

Let us consider $S, x$. We say that $x$ is $S$-null if and only if:

(Def. 17) Not contradiction.

Let us consider $S, D$ and let $P_{1}$ be a set. Then $\left(P_{1}, D\right)$-termEq is a binary relation on AllTerms Of $S$.

Let us consider $S, p_{2}$ and let $P_{2}, P_{3}$ be finite subsets of AllFormulasOf $S$. One can check that $\left\langle P_{2} \cup P_{3}, p_{2}\right\rangle$ is $S$-sequent-like.

Let us consider $S$, let $x$ be an empty set, and let $p_{2}$ be a w.f.f. string of $S$. Then $\left\langle x, p_{2}\right\rangle$ is an element of $S$-sequents.

Let us consider $S$. Note that $\emptyset \cap S$ is $S$-sequents-like.

Let us consider $S$. One can verify that there exists a set which is $S$-null.

Let us consider $S$. One can check that every set which is $S$-sequent-like is also $S$-null.

Let us consider $S$. One can check that every element of $S$-sequents is $S$-null.

Let us consider $m, S, D, X$. One can verify that $(m, D)$-derivables $(X)$ is $S$-sequents-like.

Let us consider $S, Y$ and let $X$ be an $S$-sequents-like set. One can verify that $X \cap Y$ is $S$-sequents-like.

Let us consider $S, D, m, X$. Note that every set which is $(m, X, D)$-derivable is also $S$-sequent-like.

Let us consider $S, D$ and let $P_{2}, P_{3}$ be sets. Observe that every set which is $\left(P_{2} \backslash P_{3}, D\right)$-provable is also $\left(P_{2}, D\right)$-provable.

Let us consider $S, D$ and let $P_{2}, P_{3}$ be sets. Observe that every set which is $\left(P_{2} \backslash P_{3}, D\right)$-provable is also $\left(P_{2} \cup P_{3}, D\right)$-provable.

Let us consider $S, D$ and let $P_{2}, P_{3}$ be sets. Observe that every set which is $\left(P_{2} \cap P_{3}, D\right)$-provable is also $\left(P_{2}, D\right)$-provable.

Let us consider $S, D$, let $X$ be a set, and let $x$ be a subset of $X$. Note that every set which is $(x, D)$-provable is also $(X, D)$-provable.

Let us consider $S$, let $p_{5}$ be a finite subset of AllFormulasOf $S$, and let $p_{2}$ be a w.f.f. string of $S$. One can check that $\left\langle p_{5}, p_{2}\right\rangle$ is $S$-sequent-like.

Let us consider $S$ and let $p_{3}, p_{4}$ be w.f.f. strings of $S$. Observe that $\left\langle\left\{p_{3}\right\}\right.$, $\left.p_{4}\right\rangle$ is $S$-sequent-like. Let $p_{6}$ be a w.f.f. string of $S$. Note that $\left\langle\left\{p_{3}, p_{4}\right\}, p_{6}\right\rangle$ is $S$-sequent-like. 
Let us consider $S, p_{3}, p_{4}$ and let $P_{1}$ be a finite subset of AllFormulasOf $S$. One can verify that $\left\langle P_{1} \cup\left\{p_{3}\right\}, p_{4}\right\rangle$ is $S$-sequent-like.

Let us consider $S, D$. Note that there exists a subset of AllFormulasOf $S$ which is $D$-expanded.

Let us consider $S, D$. Observe that there exists a set which is $D$-expanded.

Let $S_{1}$ be a set, let $S$ be a language, and let $s_{1}$ be an $S$-null set. We say that $s_{1}$ rule $0 S_{1}$ if and only if:

(Def. 18) $\quad\left(s_{1}\right)_{\mathbf{2}} \in\left(s_{1}\right)_{\mathbf{1}}$.

We say that $s_{1}$ rule $1 S_{1}$ if and only if:

(Def. 19) There exists a set $y$ such that $y \in S_{1}$ and $y_{\mathbf{1}} \subseteq\left(s_{1}\right)_{\mathbf{1}}$ and $\left(s_{1}\right)_{\mathbf{2}}=y_{\mathbf{2}}$.

We say that $s_{1}$ rule $2 S_{1}$ if and only if:

(Def. 20) $\left(s_{1}\right)_{1}$ is empty and there exists a termal string $t$ of $S$ such that $\left(s_{1}\right)_{2}=$ $\langle$ TheEqSymbOf $S\rangle\urcorner t^{\frown} t$.

We say that $s_{1}$ rule $3 \mathrm{a} S_{1}$ if and only if the condition (Def. 21) is satisfied.

(Def. 21) There exist termal strings $t, t_{1}, t_{2}$ of $S$ and there exists a set $x$ such that $x \in S_{1}$ and $\left(s_{1}\right)_{1}=x_{1} \cup\{\langle$ TheEqSymbOf $\left.S\rangle\urcorner t_{1} \frown t_{2}\right\}$ and $x_{\mathbf{2}}=$ $\langle$ TheEqSymbOf $S\rangle\urcorner t^{\frown} t_{1}$ and $\left(s_{1}\right)_{2}=\langle$ TheEqSymbOf $\left.S\rangle\right\urcorner t^{\frown} t_{2}$.

We say that $s_{1}$ rule $3 \mathrm{~b} S_{1}$ if and only if:

(Def. 22) There exist termal strings $t_{1}, t_{2}$ of $S$ such that $\left(s_{1}\right)_{1}=$ $\left\{\langle\text { TheEqSymbOf } S\urcorner^{\frown} t_{1} \frown t_{2}\right\}$ and $\left(s_{1}\right)_{2}=\langle$ TheEqSymbOf $\left.\left.S\rangle\right\urcorner t_{2}\right\urcorner t_{1}$.

We say that $s_{1}$ rule $3 \mathrm{~d} S_{1}$ if and only if the condition (Def. 23) is satisfied.

(Def. 23) There exists a low-compounding element $s$ of $S$ and there exist $|\operatorname{ar} s|$ element elements $T, U$ of (AllTermsOf $S)^{*}$ such that

(i) $s$ is operational,

(ii) $\quad\left(s_{1}\right)_{\mathbf{1}}=\left\{\langle\right.$ TheEqSymbOf $S\rangle \frown T_{1}(j) \frown U_{1}(j) ; j$ ranges over elements of Seg $\mid$ ar $s \mid, T_{1}$ ranges over functions from Seg|ar $s \mid$ into (AllSymbolsOf $\left.S\right)^{*} \backslash$ $\{\emptyset\}, U_{1}$ ranges over functions from Seg $\mid$ ar $s \mid$ into (AllSymbolsOf $\left.S\right)^{*} \backslash\{\emptyset\}$ : $\left.T_{1}=T \wedge U_{1}=U\right\}$, and

(iii) $\quad\left(s_{1}\right)_{\mathbf{2}}=\langle\text { TheEqSymbOf } S\rangle^{\frown}(s \text {-compound } T)^{\frown}(s$-compound $U)$.

We say that $s_{1}$ rule $3 \mathrm{e} S_{1}$ if and only if the condition (Def. 24) is satisfied.

(Def. 24) There exists a relational element $s$ of $S$ and there exist $\mid$ ar $s \mid$-element elements $T, U$ of (AllTermsOf $S)^{*}$ such that

(i) $\quad\left(s_{1}\right)_{1}=\{s$-compound $T\} \cup\left\{\langle\right.$ TheEqSymbOf $S\rangle \frown T_{1}(j) \frown U_{1}(j) ; j$ ranges over elements of Seg $|\operatorname{ar} s|, T_{1}$ ranges over functions from Seg $|\operatorname{ar} s|$ into (AllSymbolsOf $S)^{*} \backslash\{\emptyset\}, U_{1}$ ranges over functions from Seg $\mid$ ar $s \mid$ into (AllSymbolsOf $\left.S)^{*} \backslash\{\emptyset\}: T_{1}=T \wedge U_{1}=U\right\}$, and

(ii) $\quad\left(s_{1}\right)_{2}=s$-compound $U$.

We say that $s_{1}$ rule $4 S_{1}$ if and only if the condition (Def. 25) is satisfied. 
(Def. 25) There exists a literal element $l$ of $S$ and there exists a w.f.f. string $p_{2}$ of $S$ and there exists a termal string $t$ of $S$ such that $\left(s_{1}\right)_{1}=\left\{(l, t)\right.$ SubstIn $\left.p_{2}\right\}$ and $\left(s_{1}\right)_{2}=\langle l\rangle \frown p_{2}$.

We say that $s_{1}$ rule $5 S_{1}$ if and only if:

(Def. 26) There exist literal elements $v_{1}, v_{2}$ of $S$ and there exist $x, p$ such that $\left(s_{1}\right)_{1}=x \cup\left\{\left\langle v_{1}\right\rangle \frown p\right\}$ and $v_{2}$ is $x \cup\{p\} \cup\left\{s_{12}\right\}$-absent and $\left\langle x \cup\left\{\left(v_{1} \operatorname{SubstWith} v_{2}\right)(p)\right\},\left(s_{1}\right)_{\mathbf{2}}\right\rangle \in S_{1}$.

We say that $s_{1}$ rule $6 S_{1}$ if and only if the condition (Def. 27) is satisfied.

(Def. 27) There exist sets $y_{1}, y_{2}$ and there exist w.f.f. strings $p_{3}, p_{4}$ of $S$ such that $y_{1}, y_{2} \in S_{1}$ and $\left(y_{1}\right)_{\mathbf{1}}=\left(y_{2}\right)_{\mathbf{1}}=\left(s_{1}\right)_{\mathbf{1}}$ and $\left(y_{1}\right)_{\mathbf{2}}=$ $\langle$ TheNorSymbOf $S\rangle \frown p_{3} \frown p_{3}$ and $\left(y_{2}\right)_{2}=\langle$ TheNorSymbOf $S\rangle{ }^{\frown} p_{4} \frown p_{4}$ and $\left(s_{1}\right)_{2}=\langle$ TheNorSymbOf $S\rangle \frown p_{3} \frown p_{4}$.

We say that $s_{1}$ rule $7 S_{1}$ if and only if:

(Def. 28) There exists a set $y$ and there exist w.f.f. strings $p_{3}, p_{4}$ of $S$ such that $y \in S_{1}$ and $y_{1}=\left(s_{1}\right)_{1}$ and $y_{2}=\langle\text { TheNorSymbOf } S\rangle^{\frown} p_{3}{ }^{\frown} p_{4}$ and $\left(s_{1}\right)_{2}=$ $\langle$ TheNorSymbOf $S\rangle \frown p_{4} \frown p_{3}$.

We say that $s_{1}$ rule $8 S_{1}$ if and only if the condition (Def. 29) is satisfied.

(Def. 29) There exist sets $y_{1}, y_{2}$ and there exist w.f.f. strings $p_{2}, p_{3}, p_{4}$ of $S$ such that $y_{1}, y_{2} \in S_{1}$ and $\left(y_{1}\right)_{1}=\left(y_{2}\right)_{1}$ and $\left(y_{1}\right)_{2}=p_{3}$ and $\left(y_{2}\right)_{\mathbf{2}}=\langle$ TheNorSymbOf $S\rangle \frown p_{3} \frown p_{4}$ and $\left\{p_{2}\right\} \cup\left(s_{1}\right)_{1}=\left(y_{1}\right)_{1}$ and $\left(s_{1}\right)_{\mathbf{2}}=\langle$ TheNorSymbOf $S\rangle \frown p_{2}{ }^{\frown} p_{2}$.

We say that $s_{1}$ rule $9 S_{1}$ if and only if:

(Def. 30) There exists a set $y$ and there exists a w.f.f. string $p_{2}$ of $S$ such that $y \in S_{1}$ and $\left(s_{1}\right)_{2}=p_{2}$ and $y_{1}=\left(s_{1}\right)_{1}$ and $y_{2}=\operatorname{xnot} \operatorname{xnot} p_{2}$.

Let $S$ be a language. The functor P0 $S$ yielding a relation between $2^{S \text {-sequents }}$ and $S$-sequents is defined by:

(Def. 31) For every element $S_{1}$ of $2^{S \text {-sequents }}$ and for every element $s_{1}$ of $S$-sequents holds $\left\langle S_{1}, s_{1}\right\rangle \in \mathrm{P} 0 S$ iff $s_{1}$ rule $0 S_{1}$.

The functor P1 $S$ yields a relation between $2^{S \text {-sequents }}$ and $S$-sequents and is defined as follows:

(Def. 32) For every element $S_{1}$ of $2^{S \text {-sequents }}$ and for every element $s_{1}$ of $S$-sequents holds $\left\langle S_{1}, s_{1}\right\rangle \in \mathrm{P} 1 S$ iff $s_{1}$ rule $1 S_{1}$.

The functor P2 $S$ yields a relation between $2^{S \text {-sequents }}$ and $S$-sequents and is defined as follows:

(Def. 33) For every element $S_{1}$ of $2^{S \text {-sequents }}$ and for every element $s_{1}$ of $S$-sequents holds $\left\langle S_{1}, s_{1}\right\rangle \in \mathrm{P} 2 S$ iff $s_{1}$ rule $2 S_{1}$.

The functor P3a $S$ yielding a relation between $2^{S \text {-sequents }}$ and $S$-sequents is defined as follows: 
(Def. 34) For every element $S_{1}$ of $2^{S \text {-sequents }}$ and for every element $s_{1}$ of $S$-sequents holds $\left\langle S_{1}, s_{1}\right\rangle \in$ P3a $S$ iff $s_{1}$ rule 3 a $S_{1}$.

The functor P3b $S$ yields a relation between $2^{S \text {-sequents }}$ and $S$-sequents and is defined as follows:

(Def. 35) For every element $S_{1}$ of $2^{S \text {-sequents }}$ and for every element $s_{1}$ of $S$-sequents holds $\left\langle S_{1}, s_{1}\right\rangle \in$ P $3 \mathrm{~b} S$ iff $s_{1}$ rule $3 \mathrm{~b} S_{1}$.

The functor P3d $S$ yields a relation between $2^{S \text {-sequents }}$ and $S$-sequents and is defined as follows:

(Def. 36) For every element $S_{1}$ of $2^{S \text {-sequents }}$ and for every element $s_{1}$ of $S$-sequents holds $\left\langle S_{1}, s_{1}\right\rangle \in$ P3d $S$ iff $s_{1}$ rule $3 \mathrm{~d} S_{1}$.

The functor P3e $S$ yielding a relation between $2^{S \text {-sequents }}$ and $S$-sequents is defined by:

(Def. 37) For every element $S_{1}$ of $2^{S \text {-sequents }}$ and for every element $s_{1}$ of $S$-sequents holds $\left\langle S_{1}, s_{1}\right\rangle \in \mathrm{P} 3 \mathrm{e} S$ iff $s_{1}$ rule $3 \mathrm{e} S_{1}$.

The functor $\mathrm{P} 4 S$ yielding a relation between $2^{S \text {-sequents }}$ and $S$-sequents is defined by:

(Def. 38) For every element $S_{1}$ of $2^{S \text {-sequents }}$ and for every element $s_{1}$ of $S$-sequents holds $\left\langle S_{1}, s_{1}\right\rangle \in \mathrm{P} 4 S$ iff $s_{1}$ rule $4 S_{1}$.

The functor P5 $S$ yields a relation between $2^{S \text {-sequents }}$ and $S$-sequents and is defined by:

(Def. 39) For every element $S_{1}$ of $2^{S \text {-sequents }}$ and for every element $s_{1}$ of $S$-sequents holds $\left\langle S_{1}, s_{1}\right\rangle \in \mathrm{P} 5 S$ iff $s_{1}$ rule $5 S_{1}$.

The functor P6 $S$ yielding a relation between $2^{S \text {-sequents }}$ and $S$-sequents is defined by:

(Def. 40) For every element $S_{1}$ of $2^{S \text {-sequents }}$ and for every element $s_{1}$ of $S$-sequents holds $\left\langle S_{1}, s_{1}\right\rangle \in \mathrm{P} 6 S$ iff $s_{1}$ rule $6 S_{1}$.

The functor P7 $S$ yielding a relation between $2^{S \text {-sequents }}$ and $S$-sequents is defined as follows:

(Def. 41) For every element $S_{1}$ of $2^{S \text {-sequents }}$ and for every element $s_{1}$ of $S$-sequents holds $\left\langle S_{1}, s_{1}\right\rangle \in \mathrm{P} 7 S$ iff $s_{1}$ rule $7 S_{1}$.

The functor P8 $S$ yields a relation between $2^{S \text {-sequents }}$ and $S$-sequents and is defined as follows:

(Def. 42) For every element $S_{1}$ of $2^{S \text {-sequents }}$ and for every element $s_{1}$ of $S$-sequents holds $\left\langle S_{1}, s_{1}\right\rangle \in \mathrm{P} 8 S$ iff $s_{1}$ rule $8 S_{1}$.

The functor P9 $S$ yields a relation between $2^{S \text {-sequents }}$ and $S$-sequents and is defined as follows:

(Def. 43) For every element $S_{1}$ of $2^{S \text {-sequents }}$ and for every element $s_{1}$ of $S$-sequents holds $\left\langle S_{1}, s_{1}\right\rangle \in \mathrm{P} 9 S$ iff $s_{1}$ rule $9 S_{1}$. 
Let us consider $S$ and let $R$ be a relation between $2^{S \text {-sequents }}$ and $S$-sequents. The functor FuncRule $R$ yields a rule of $S$ and is defined by:

(Def. 44) For every set $i_{1}$ such that $i_{1} \in 2^{S \text {-sequents }}$ holds (FuncRule $\left.R\right)\left(i_{1}\right)=\{x \in$ $S$-sequents: $\left.\left\langle i_{1}, x\right\rangle \in R\right\}$.

Let us consider $S$. The functor R0 $S$ yielding a rule of $S$ is defined as follows:

(Def. 45) R0 $S=$ FuncRule P0 $S$.

The functor R1 $S$ yielding a rule of $S$ is defined as follows:

(Def. 46) R1 $S=$ FuncRule P1 $S$.

The functor R2 $S$ yielding a rule of $S$ is defined by:

(Def. 47) R2 $S=$ FuncRule P2 $S$.

The functor R3a $S$ yielding a rule of $S$ is defined by:

(Def. 48) R3a $S=$ FuncRule P3a $S$.

The functor R3b $S$ yielding a rule of $S$ is defined as follows:

(Def. 49) R3b $S=$ FuncRule P3b $S$.

The functor R3d $S$ yielding a rule of $S$ is defined as follows:

(Def. 50) R3d $S=$ FuncRule P3d $S$.

The functor R3e $S$ yielding a rule of $S$ is defined by:

(Def. 51) R3e $S=$ FuncRule P3e $S$.

The functor R4 $S$ yields a rule of $S$ and is defined as follows:

(Def. 52) R4 $S=$ FuncRule P4 $S$.

The functor R5 $S$ yielding a rule of $S$ is defined as follows:

(Def. 53) R5 $S=$ FuncRule P5 $S$.

The functor R6 $S$ yields a rule of $S$ and is defined by:

(Def. 54) R6 $S=$ FuncRule P6 $S$.

The functor R7 $S$ yields a rule of $S$ and is defined by:

(Def. 55) $\quad$ R7 $S=$ FuncRule P7 $S$.

The functor R8 $S$ yielding a rule of $S$ is defined as follows:

(Def. 56) R8 $S=$ FuncRule P8 $S$.

The functor R9 $S$ yields a rule of $S$ and is defined by:

(Def. 57) R9 $S=$ FuncRule P9 $S$.

Let us consider $S$ and let $t$ be a termal string of $S$.

Note that $\left\{\left\langle\emptyset,\langle\text { TheEqSymbOf } S\rangle^{\frown} t^{\frown} t\right\rangle\right\}$ is $\{\mathrm{R} 2 S\}$-derivable. Note that $\mathrm{R} 2 S$ is isotone. One can verify that R3b $S$ is isotone.

Let $t, t_{1}, t_{2}$ be termal strings of $S$, and let $p_{5}$ be a finite subset of

AllFormulasOf $S$. Observe that $\left\langle p_{5} \cup\{\langle\right.$ TheEqSymbOf $\left.S\rangle\urcorner t_{1} t_{2}\right\}$,

$\left.\langle\text { TheEqSymbOf } S\rangle^{\frown} t^{\frown} t_{2}\right\rangle$ is $\left(1,\left\{\left\langle p_{5},\langle\text { TheEqSymbOf } S\rangle^{\frown} t^{\frown} t_{1}\right\rangle\right\},\{\right.$ R3a $\left.S\}\right)$ derivable. 
Let us consider $S$, let $t, t_{1}, t_{2}$ be termal strings of $S$, and let $p_{2}$ be a w.f.f. string of $S$. Note that $\left\langle\left\{p_{2},\langle\text { TheEqSymbOf } S\rangle^{\frown} t_{1} \frown t_{2}\right\},\langle\text { TheEqSymbOf } S\rangle^{\frown}\right.$ $\left.t^{\frown} t_{2}\right\rangle$ is $\left(1,\left\{\left\langle\left\{p_{2}\right\}\right.\right.\right.$, $\langle$ TheEqSymbOf $\left.\left.S\rangle \frown t^{\frown} t_{1}\right\rangle\right\},\{$ R3a $\left.S\}\right)$-derivable.

Let us consider $S$, let $p_{2}$ be a w.f.f. string of $S$, and let $P_{1}$ be a finite subset of AllFormulasOf $S$. One can verify that $\left\langle P_{1} \cup\left\{p_{2}\right\}, p_{2}\right\rangle$ is $(1, \emptyset,\{\mathrm{R} 0 S\})$-derivable.

Let us consider $S$ and let $p_{3}, p_{4}$ be w.f.f. strings of $S$. One can check that $\left\langle\left\{p_{3}, p_{4}\right\}, p_{3}\right\rangle$ is $(1, \emptyset,\{\mathrm{R} 0 S\})$-derivable.

Let us consider $S, p_{2}$. Note that $\left\langle\left\{p_{2}\right\}, p_{2}\right\rangle$ is $(1, \emptyset,\{\mathrm{R} 0 S\})$-derivable.

Let us consider $S$ and let $p_{2}$ be a w.f.f. string of $S$. Observe that $\left\{\left\langle\left\{p_{2}\right\}\right.\right.$, $\left.\left.p_{2}\right\rangle\right\}$ is $(\emptyset,\{\mathrm{R} 0 S\})$-derivable.

Let us consider $S$. One can verify the following observations:

* R0 $S$ is isotone,

* R3a $S$ is isotone,

* $\operatorname{R} 3 \mathrm{~d} S$ is isotone, and

* R3e $S$ is isotone.

Let us consider $K_{1}, K_{2}$. One can verify that $K_{1} \cup K_{2}$ is isotone.

Let us consider $S$ and let $t_{1}, t_{2}$ be termal strings of $S$.

Observe that $\langle$ TheEqSymbOf $\left.S\rangle\urcorner t_{1}\right\urcorner t_{2}$ is 0-w.f.f..

Let us consider $S$, let $m$ be a non zero natural number, and let $T, U$ be $m$ element elements of (AllTermsOf $S)^{*}$. The functor PairWiseEq $(T, U)$ is defined by the condition (Def. 58).

(Def. 58) PairWiseEq $(T, U)=\left\{\langle\right.$ TheEqSymbOf $S\rangle \frown T_{1}(j) \frown U_{1}(j) ; j$ ranges over elements of $\operatorname{Seg} m, T_{1}$ ranges over functions from $\operatorname{Seg} m$ into $(\text { AllSymbolsOf } S)^{*} \backslash\{\emptyset\}, U_{1}$ ranges over functions from Seg $m$ into $\left.(\text { AllSymbolsOf } S)^{*} \backslash\{\emptyset\}: T_{1}=T \wedge U_{1}=U\right\}$.

Let us consider $S$, let $m$ be a non zero natural number, and let $T_{2}, T_{3}$ be $m$-element elements of (AllTermsOf $S)^{*}$. Then PairWiseEq $\left(T_{2}, T_{3}\right)$ is a subset of AllFormulasOf $S$.

Let us consider $S$, let $m$ be a non zero natural number, and let $T, U$ be $m$ element elements of (AllTermsOf $S)^{*}$. Observe that PairWiseEq $(T, U)$ is finite.

Let us consider $S$, let $s$ be a relational element of $S$, and let $T_{2}, T_{3}$ be $|\operatorname{ar} s|$ element elements of (AllTermsOf $S)^{*}$. Observe that $\left\{\left\langle\operatorname{PairWiseEq}\left(T_{2}, T_{3}\right) \cup\right.\right.$ $\left\{s\right.$-compound $\left.T_{2}\right\}, s$-compound $\left.\left.T_{3}\right\rangle\right\}$ is $(\emptyset,\{\mathrm{R} 3 \mathrm{e} S\})$-derivable.

Let us consider $m, S, D$. We say that $D$ is $m$-ranked if and only if:

(Def. 59)(i) R0 $S$, R2 $S$, R3a $S$, R3b $S \in D$ if $m=0$,

(ii) R0 $S, \mathrm{R} 2 S, \mathrm{R} 3 \mathrm{a} S, \mathrm{R} 3 \mathrm{~b} S, \mathrm{R} 3 \mathrm{~d} S, \mathrm{R} 3 \mathrm{e} S \in D$ if $m=1$,

(iii) $\mathrm{R} 0 S, \mathrm{R} 1 S, \mathrm{R} 2 S, \mathrm{R} 3 \mathrm{a} S, \mathrm{R} 3 \mathrm{~b} S, \mathrm{R} 3 \mathrm{~d} S, \mathrm{R} 3 \mathrm{e} S, \mathrm{R} 4 S, \mathrm{R} 5 S, \mathrm{R} 6 S, \mathrm{R} 7 S$, $\mathrm{R} 8 S \in D$ if $m=2$,

(iv) $D=\emptyset$, otherwise. 
Let us consider $S$. One can verify that every rule set of $S$ which is 1-ranked is also 0-ranked and every rule set of $S$ which is 2-ranked is also 1-ranked.

Let us consider $S$. The functor $S$-rules yields a rule set of $S$ and is defined by:

(Def. 60) $S$-rules $=\{\mathrm{R} 0 S, \mathrm{R} 1 S, \mathrm{R} 2 S, \mathrm{R} 3 \mathrm{a} S, \mathrm{R} 3 \mathrm{~b} S, \mathrm{R} 3 \mathrm{~d} S, \mathrm{R} 3 \mathrm{e} S, \mathrm{R} 4 S\} \cup$ $\{\mathrm{R} 5 S, \mathrm{R} 6 S, \mathrm{R} 7 S, \mathrm{R} 8 S\}$.

Let us consider $S$. Observe that $S$-rules is 2-ranked.

Let us consider $S$. Note that there exists a rule set of $S$ which is 2-ranked.

Let us consider $S$. Observe that there exists a rule set of $S$ which is 1-ranked.

Let us consider $S$. Note that there exists a rule set of $S$ which is 0-ranked.

Let us consider $S$, let $D$ be a 1-ranked rule set of $S$, let $X$ be a $D$-expanded set, and let us consider $a$. Observe that $X$-freeInterpreter $a$ is $(X, D)$-termEqrespecting.

Let us consider $S$, let $D$ be a 0 -ranked rule set of $S$, and let $X$ be a $D$ expanded set. Observe that $(X, D)$-termEq is total, symmetric, and transitive.

Let us consider $S$. Observe that there exists a 0-ranked rule set of $S$ which is 1-ranked.

The following proposition is true

(1) If $D_{1} \subseteq D_{2}$ and if $D_{2}$ is isotone or $D_{1}$ is isotone and if $Y$ is $\left(X, D_{1}\right)$ derivable, then $Y$ is $\left(X, D_{2}\right)$-derivable.

Let us consider $S, S_{6}$. One can verify that $\left\{S_{6}\right\}$ is $S$-sequents-like.

Let us consider $S, S_{11}, S_{5}$. One can check that $S_{11} \cup S_{5}$ is $S$-sequents-like.

Let us consider $S$ and let $x, y$ be $S$-sequent-like sets. Observe that $\{x, y\}$ is $S$-sequents-like.

Let us consider $S, p_{3}, p_{4}$. Note that $\left\langle\left\{\operatorname{xnot} p_{3}, \operatorname{xnot} p_{4}\right\},\langle\text { TheNorSymbOf } S\rangle^{\frown}\right.$ $\left.p_{3}{ }^{\frown} p_{4}\right\rangle$ is $\left(1,\left\{\left\langle\left\{\operatorname{xnot} p_{3}, \operatorname{xnot} p_{4}\right\}, \operatorname{xnot} p_{3}\right\rangle,\left\langle\left\{\operatorname{xnot} p_{3}, \operatorname{xnot} p_{4}\right\}, \operatorname{xnot} p_{4}\right\rangle\right\},\{\operatorname{R} 6 S\}\right)-$ derivable.

Let us consider $S, p_{3}, p_{4}$. One can check that $\left\langle\left\{p_{3}, p_{4}\right\}, p_{4}\right\rangle$ is $(1, \emptyset,\{\operatorname{R} 0 S\})$ derivable.

We now state two propositions:

(2) For every relation $R$ between $2^{S \text {-sequents }}$ and $S$-sequents such that $\left\langle S_{4}\right.$, $\left.S_{6}\right\rangle \in R$ holds $S_{6} \in($ FuncRule $R)\left(S_{4}\right)$.

(3) If $x \in R(X)$, then $x$ is $(1, X,\{R\})$-derivable.

Let us consider $S, D, X$. Let us observe that $X$ is $D$-expanded if and only if:

(Def. 61) If $x$ is $(X, D)$-provable, then $x \in X$.

The following four propositions are true:

(4) If $p_{2} \in X$, then $p_{2}$ is $(X,\{\mathrm{R} 0 S\})$-provable.

(5) Suppose that

(i) $D_{1} \cup D_{2}$ is isotone, 
(ii) $D_{1} \cup D_{2} \cup D_{3}$ is isotone,

(iii) $\quad x$ is $\left(m, S_{11}, D_{1}\right)$-derivable,

(iv) $\quad y$ is $\left(m, S_{5}, D_{2}\right)$-derivable, and

(v) $z$ is $\left(n,\{x, y\}, D_{3}\right)$-derivable.

Then $z$ is $\left(m+n, S_{11} \cup S_{5}, D_{1} \cup D_{2} \cup D_{3}\right)$-derivable.

(6) Suppose $D_{1}$ is isotone and $D_{1} \cup D_{2}$ is isotone and $y$ is $\left(m, X, D_{1}\right)$ derivable and $z$ is $\left(n,\{y\}, D_{2}\right)$-derivable. Then $z$ is $\left(m+n, X, D_{1} \cup D_{2}\right)$ derivable.

(7) If $x$ is $(m, X, D)$-derivable, then $\{x\}$ is $(X, D)$-derivable.

Let us consider $S$. Observe that R6 $S$ is isotone.

One can prove the following propositions:

(8) If $D_{1} \subseteq D_{2}$ and if $D_{1}$ is isotone or $D_{2}$ is isotone and if $x$ is $\left(X, D_{1}\right)$ provable, then $x$ is $\left(X, D_{2}\right)$-provable.

(9) If $X \subseteq Y$ and $x$ is $(X, D)$-provable, then $x$ is $(Y, D)$-provable.

Let us consider $S$. Note that R 8 is isotone.

Let us consider $S$. Observe that $\mathrm{R} 1 S$ is isotone.

Next we state the proposition

(10) If $\{y\}$ is $\left(S_{4}, D\right)$-derivable, then there exists $m_{1}$ such that $y$ is $\left(m_{1}, S_{4}, D\right)$ derivable.

Let us consider $S, D, X$. Observe that every set which is $(X, D)$-derivable is also $S$-sequents-like.

Let us consider $S, D, X, x$. Let us observe that $x$ is $(X, D)$-provable if and only if:

(Def. 62) There exists a set $H$ and there exists $m$ such that $H \subseteq X$ and $\langle H, x\rangle$ is $(m, \emptyset, D)$-derivable.

The following proposition is true

(11) If $D_{1} \subseteq D_{2}$ and if $D_{2}$ is isotone or $D_{1}$ is isotone and if $x$ is $\left(m, X, D_{1}\right)$ derivable, then $x$ is $\left(m, X, D_{2}\right)$-derivable.

Let us consider $S$. Observe that R7 $S$ is isotone.

Next we state the proposition

(12) If $x$ is $(X, D)$-provable, then $x$ is a w.f.f. string of $S$.

In the sequel $F$ denotes a rule set of $S$.

Let us consider $S, D_{1}$ and let $X$ be a $D_{1}$-expanded set. One can verify that $(S, X)$-freeInterpreter is $\left(X, D_{1}\right)$-termEq-respecting.

Let us consider $S$, let $D$ be a 0 -ranked rule set of $S$, and let $X$ be a $D$ expanded set. The functor $D$ Henkin $X$ yielding a function is defined by:

(Def. 63) $D$ Henkin $X=(S, X)$-freeInterpreter quotient $(X, D)$-termEq .

Let us consider $S$, let $D$ be a 0 -ranked rule set of $S$, and let $X$ be a $D$ expanded set. One can check that $D$ Henkin $X$ is OwnSymbolsOf $S$-defined. 
Let us consider $S, D_{1}$ and let $X$ be a $D_{1}$-expanded set. Observe that $D_{1}$ Henkin $X$ is $\left(S\right.$, Classes $\left(X, D_{1}\right)$-termEq)-interpreter-like.

Let us consider $S, D_{1}$ and let $X$ be a $D_{1}$-expanded set. Then $D_{1}$ Henkin $X$ is an element of Classes $\left(\left(X, D_{1}\right)\right.$-termEq)-InterpretersOf $S$.

Let us consider $S, p_{3}, p_{4}$. One can verify that $\langle\text { TheNorSymbOf } S\rangle^{\frown} p_{3}{ }^{\wedge} p_{4}$ is $\left(\left\{\operatorname{xnot} p_{3}, \operatorname{xnot} p_{4}\right\},\{\operatorname{R} 0 S\} \cup\{\operatorname{R} 6 S\}\right)$-provable.

Let us consider $S$. Note that every 0-ranked rule set of $S$ is non empty.

Let us consider $S, x$. We say that $x$ is $S$-premises-like if and only if:

(Def. 64) $x \subseteq$ AllFormulasOf $S$ and $x$ is finite.

Let us consider $S$. One can verify that every set which is $S$-premises-like is also finite.

Let us consider $S, p_{2}$. Note that $\left\{p_{2}\right\}$ is $S$-premises-like.

Let us consider $S$ and let $e$ be an empty set. One can check that $e$ null $S$ is $S$-premises-like.

Let us consider $X, S$. Observe that there exists a subset of $X$ which is $S$-premises-like.

Let us consider $S$. Observe that there exists a set which is $S$-premises-like.

Let us consider $S$ and let $X$ be an $S$-premises-like set. Observe that every subset of $X$ is $S$-premises-like.

In the sequel $H_{3}$ denotes an $S$-premises-like set.

Let us consider $S, H_{2}, H_{1}$. Then $H_{1}$ null $H_{2}$ is a subset of AllFormulasOf $S$.

Let us consider $S, H, x$. Note that $H$ null $x$ is $S$-premises-like.

Let us consider $S, H_{1}, H_{2}$. Note that $H_{1} \cup H_{2}$ is $S$-premises-like.

Let us consider $S, H, p_{2}$. Observe that $\left\langle H, p_{2}\right\rangle$ is $S$-sequent-like.

Let us consider $S, H_{1}, H_{2}, p_{2}$. One can verify that $\left\langle H_{1} \cup H_{2}, p_{2}\right\rangle$ is $\left(1,\left\{\left\langle H_{1}\right.\right.\right.$, $\left.\left.\left.p_{2}\right\rangle\right\},\{\mathrm{R} 1 S\}\right)$-derivable.

Let us consider $S, H, p_{2}, p_{3}, p_{4}$. One can check that $\left\langle H\right.$ null $p_{3}{ }^{\frown} p_{4}$, $\left.\operatorname{xnot} p_{2}\right\rangle$ is (1, $\left\{\left\langle H \cup\left\{p_{2}\right\}, p_{3}\right\rangle,\left\langle H \cup\left\{p_{2}\right\},\langle\right.\right.$ TheNorSymbOf $\left.\left.\left.S\rangle{ }^{\frown} p_{3}{ }^{\frown} p_{4}\right\rangle\right\},\{\mathrm{R} 8 S\}\right)$-derivable.

Let us consider $S$. One can verify that $\emptyset$ null $S$ is $S$-sequents-like.

Let us consider $S, H, p_{2}$. Observe that $\left\langle H \cup\left\{p_{2}\right\}, p_{2}\right\rangle$ is $(1, \emptyset,\{\operatorname{R} 0 S\})$ derivable. Let us consider $p_{3}, p_{4}$. Note that $\left\langle H\right.$ null $p_{4}$, $\left.\operatorname{xnot} p_{3}\right\rangle$ is

$\left(2,\left\{\left\langle H,\langle\text { TheNorSymbOf } S\rangle^{\frown} p_{3}{ }^{\wedge} p_{4}\right\rangle\right\},\{\mathrm{R} 0 S\} \cup\{\mathrm{R} 1 S\} \cup\{\mathrm{R} 8 S\}\right)$-derivable.

Let us consider $S, H, p_{3}, p_{4}$. Note that $\left\langle H\right.$, $\left.\langle\text { TheNorSymbOf } S\rangle^{\frown} p_{4}{ }^{\frown} p_{3}\right\rangle$ is $\left(1,\left\{\langle H,\langle\right.\right.$ TheNorSymbOf $\left.\left.\left.S\rangle\urcorner p_{3} \frown p_{4}\right\rangle\right\},\{\mathrm{R} 7 S\}\right)$-derivable.

Let us consider $S, H, p_{3}, p_{4}$. Observe that $\left\langle H\right.$ null $\left.p_{3}, \operatorname{xnot} p_{4}\right\rangle$ is $(3,\{\langle H$, $\langle$ TheNorSymbOf $\left.\left.\left.S\rangle\urcorner p_{3} \frown p_{4}\right\rangle\right\},\{\operatorname{R} 0 S\} \cup\{\mathrm{R} 1 S\} \cup\{\mathrm{R} 8 S\} \cup\{\mathrm{R} 7 S\}\right)$-derivable.

Let us consider $S, S_{6}$. Observe that $\left(S_{6}\right)_{1}$ is $S$-premises-like.

Let us consider $S, X, D$. Then $D$ null $X$ is a rule set of $S$.

Let us consider $S, p_{3}, p_{4}, l_{1}, H$ and let $l_{2}$ be an $H \cup\left\{p_{3}\right\} \cup\left\{p_{4}\right\}$-absent literal element of $S$. 
Note that $\left\langle\left(H \cup\left\{\left\langle l_{1}\right\urcorner^{\frown} p_{3}\right\}\right)\right.$ null $\left.l_{2}, p_{4}\right\rangle$ is $\left(1,\left\{\left\langle H \cup\left\{\left(l_{1}, l_{2}\right)\right.\right.\right.\right.$-SymbolSubstIn $\left.p_{3}\right\}$, $\left.\left.\left.p_{4}\right\rangle\right\},\{\operatorname{R} 5 S\}\right)$-derivable.

Let us consider $S, D, X$. We say that $X$ is $D$-inconsistent if and only if:

(Def. 65) There exist $p_{3}, p_{4}$ such that $p_{3}$ is $(X, D)$-provable and $\langle\text { TheNorSymbOf } S\rangle^{\frown}$ $p_{3} \frown p_{4}$ is $(X, D)$-provable.

Let us consider $m_{2}, S, H_{1}, H_{2}, p_{2}$. Note that $\left\langle\left(H_{1} \cup H_{2}\right)\right.$ null $\left.m_{2}, p_{2}\right\rangle$ is $\left(m_{2},\left\{\left\langle H_{1}, p_{2}\right\rangle\right\},\{\mathrm{R} 1 S\}\right)$-derivable.

Let us consider $S$. Observe that there exists an isotone rule set of $S$ which is non empty.

We now state the proposition

(13) If $X$ is $D$-inconsistent and $D$ is isotone and $\mathrm{R} 1 S, \mathrm{R} 8 S \in D$, then $\operatorname{xnot} p_{2}$ is $(X, D)$-provable.

Let us consider $S$. Observe that R5 $S$ is isotone.

Let us consider $S, l, t, p_{2}$. Observe that $\left\langle\left\{(l, t) \operatorname{SubstIn} p_{2}\right\},\langle l\rangle \frown p_{2}\right\rangle$ is $(1, \emptyset,\{\mathrm{R} 4 S\})$-derivable.

Let us consider $S$. One can verify that $\mathrm{R} 4 S$ is isotone.

Let us consider $S, X$. We say that $X$ is $S$-witnessed if and only if:

(Def. 66) For all $l_{1}, p_{3}$ such that $\left\langle l_{1}\right\rangle \frown p_{3} \in X$ there exists $l_{2}$ such that $\left(l_{1}, l_{2}\right)$-SymbolSubstIn $p_{3} \in X$ and $l_{2} \notin \operatorname{rng} p_{3}$.

We now state the proposition

$(14)^{3}$ Let $X$ be a $D_{1}$-expanded set. Suppose R1S, R4S, R6S, R7 $S, \operatorname{R} 8 S \in D_{1}$ and $X$ is $S$-mincover and $S$-witnessed. Then $\left(D_{1}\right.$ Henkin $\left.X\right)$-TruthEval $p_{1}=1$ if and only if $p_{1} \in X$.

Let us consider $S, D, X$. We introduce $X$ is $D$-consistent as an antonym of $X$ is $D$-inconsistent.

We now state the proposition

(15) For every subset $X$ of $Y$ such that $X$ is $D$-inconsistent holds $Y$ is $D$ inconsistent.

Let us consider $S, D$, let $X$ be a functional set, and let $p_{2}$ be an element of ExFormulasOf $S$. The functor $\left(D, p_{2}\right)$ AddAsWitnessTo $X$ is defined by:

(Def. 67)

$$
\left\{\begin{array}{l}
X \cup\left\{\left(S \text {-firstChar }\left(p_{2}\right),\right.\right. \text { the element } \\
\text { of LettersOf } S \backslash \text { SymbolsOf } \\
\left(\left((\text { AllSymbolsOf } S)^{*} \backslash\{\emptyset\}\right) \cap(X \cup\right. \\
\left.\left.\left.\left.\left\{\text { head } p_{2}\right\}\right)\right)\right) \text {-SymbolSubstIn head } p_{2}\right\}, \\
\text { if } X \cup\left\{p_{2}\right\} \text { is } D \text {-consistent and } \\
\text { LettersOf } S \backslash \text { SymbolsOf }(((\text { AllSym- } \\
\text { bolsOf } \left.\left.S)^{*} \backslash\{\emptyset\}\right) \cap\left(X \cup\left\{\text { head } p_{2}\right\}\right)\right) \neq \emptyset, \\
X, \text { otherwise. }
\end{array}\right.
$$

\footnotetext{
${ }^{3}$ Henkin's Theorem
} 
Let us consider $S, D$, let $X$ be a functional set, and let $p_{2}$ be an element of ExFormulasOf $S$. One can check that $X \backslash\left(\left(D, p_{2}\right)\right.$ AddAsWitnessTo $\left.X\right)$ is empty.

Let us consider $S, D$, let $X$ be a functional set, and let $p_{2}$ be an element of ExFormulasOf $S$. One can check that $\left(\left(D, p_{2}\right)\right.$ AddAsWitnessTo $\left.X\right) \backslash X$ is trivial.

Let us consider $S, D$, let $X$ be a functional set, and let $p_{2}$ be an element of ExFormulasOf $S$. Then $\left(D, p_{2}\right)$ AddAsWitnessTo $X$ is a subset of $X \cup$ AllFormulasOf $S$.

Let us consider $S, D$. We say that $D$ is correct if and only if the condition (Def. 68) is satisfied.

(Def. 68) Let given $p_{2}, X$. Suppose $p_{2}$ is $(X, D)$-provable. Let given $U$ and $I$ be an element of $U$-InterpretersOf $S$. If $X$ is $I$-satisfied, then $I$-TruthEval $p_{2}=1$.

Let us consider $S, t_{1}, t_{2}$. One can check that SubTerms( $\langle$ TheEqSymbOf $\left.S\rangle\right\urcorner$ $\left.t_{1} \frown t_{2}\right)-\left\langle t_{1}, t_{2}\right\rangle$ is empty.

Let us consider $S$ and let $R$ be a rule of $S$. We say that $R$ is correct if and only if:

(Def. 69) If $X$ is $S$-correct, then $R(X)$ is $S$-correct.

Let us consider $S$. Observe that every set which is $S$-sequent-like is also $S$-null.

Let us consider $S$. Note that R0 $S$ is correct.

Let us consider $S$. Note that there exists a rule of $S$ which is correct.

Let us consider $S$. One can check that R $1 S$ is correct.

Let us consider $S$. Note that R2 $S$ is correct.

Let us consider $S$. One can check that R3a $S$ is correct.

Let us consider $S$. Observe that R3b $S$ is correct.

Let us consider $S$. Observe that R3d $S$ is correct.

Let us consider $S$. Note that R3e $S$ is correct.

Let us consider $S$. One can check that R4 $S$ is correct.

Let us consider $S$. One can check that R5 $S$ is correct.

Let us consider $S$. One can verify that R6 $S$ is correct.

Let us consider $S$. Observe that R7 $S$ is correct.

Let us consider $S$. Observe that $\mathrm{R} 8 S$ is correct.

Next we state the proposition

(16) If for every rule $R$ of $S$ such that $R \in D$ holds $R$ is correct, then $D$ is correct.

Let us consider $S$ and let $R$ be a correct rule of $S$. Note that $\{R\}$ is correct. Observe that $S$-rules is correct. One can check that R9 $S$ is isotone. Let us consider $H, p_{2}$. Observe that $\left\langle H, p_{2}\right\rangle$ null 1 is $\left(1,\left\{\left\langle H, \operatorname{xnot} \operatorname{xnot} p_{2}\right\rangle\right\},\{\mathrm{R} 9 S\}\right)$ derivable.

Let us consider $X, S$. Observe that there exists an 0-w.f.f. string of $S$ which is $X$-implied. 
Let us consider $X, S$. Observe that there exists a w.f.f. string of $S$ which is $X$-implied.

Let us consider $S, X$ and let $p_{2}$ be an $X$-implied w.f.f. string of $S$. Observe that $\operatorname{xnot} \operatorname{xnot} p_{2}$ is $X$-implied.

Let us consider $X, S, p_{2}$. We say that $p_{2}$ is $X$-provable if and only if:

(Def. 70$) \quad p_{2}$ is $(X,\{\mathrm{R} 9 S\} \cup S$-rules)-provable.

\section{Constructions for Countable Languages: Witness Adjoining}

Let $X$ be a functional set, let us consider $S, D$, and let $n_{1}$ be a function from $\mathbb{N}$ into ExFormulasOf $S$. The functor $\left(D, n_{1}\right)$ AddWitnessesTo $X$ yields a function from $\mathbb{N}$ into $2^{X \cup A l l F o r m u l a s O f ~} S$ and is defined by:

(Def. 71) $\quad\left(\left(D, n_{1}\right)\right.$ AddWitnessesTo $\left.X\right)(0)=X$ and

for every $m_{1}$ holds $\left(\left(D, n_{1}\right)\right.$ AddWitnessesTo $\left.X\right)\left(m_{1}+1\right)=$ $\left(D, n_{1}\left(m_{1}\right)\right)$ AddAsWitnessTo $\left(\left(D, n_{1}\right)\right.$ AddWitnessesTo $\left.X\right)\left(m_{1}\right)$.

Let $X$ be a functional set, let us consider $S, D$, and let $n_{1}$ be a function from $\mathbb{N}$ into ExFormulasOf $S$. We introduce $\left(D, n_{1}\right)$ addw $X$ as a synonym of $\left(D, n_{1}\right)$ AddWitnessesTo $X$.

We now state the proposition

(17) Let $X$ be a functional set and $n_{1}$ be a function from $\mathbb{N}$ into ExFormulasOf $S$. Suppose $D$ is isotone and R1 $S, \mathrm{R} 8 S, \mathrm{R} 2 S, \mathrm{R} 5 S \in D$ and LettersOf $S \backslash$ SymbolsOf $\left(X \cap\left((\text { AllSymbolsOf } S)^{*} \backslash\{\emptyset\}\right)\right)$ is infinite and $X$ is $D$-consistent. Then $\left(\left(D, n_{1}\right)\right.$ addw $\left.X\right)(k) \subseteq\left(\left(D, n_{1}\right)\right.$ addw $\left.X\right)(k+m)$ and LettersOf $S \backslash$ SymbolsOf $\left(\left(\left(D, n_{1}\right)\right.\right.$ addw $\left.X\right)(m) \cap\left((\text { AllSymbolsOf } S)^{*} \backslash\right.$ $\{\emptyset\}))$ is infinite and $\left(\left(D, n_{1}\right)\right.$ addw $\left.X\right)(m)$ is $D$-consistent.

Let $X$ be a functional set, let us consider $S, D$, and let $n_{1}$ be a function from $\mathbb{N}$ into ExFormulasOf $S$. The functor $X$ WithWitnessesFrom $\left(D, n_{1}\right)$ yielding a subset of $X \cup$ AllFormulas $O$ is defined by:

(Def. 72) $X$ WithWitnessesFrom $\left(D, n_{1}\right)=\bigcup \operatorname{rng}\left(\left(D, n_{1}\right)\right.$ AddWitnessesTo $\left.X\right)$.

Let $X$ be a functional set, let us consider $S, D$, and let $n_{1}$ be a function from $\mathbb{N}$ into ExFormulasOf $S$. We introduce $X \operatorname{addw}\left(D, n_{1}\right)$ as a synonym of $X$ WithWitnessesFrom $\left(D, n_{1}\right)$.

Let $X$ be a functional set, let us consider $S, D$, and let $n_{1}$ be a function from $\mathbb{N}$ into ExFormulasOf $S$. One can verify that $X \backslash\left(X \operatorname{addw}\left(D, n_{1}\right)\right)$ is empty.

The following proposition is true

(18) Let $X$ be a functional set and $n_{1}$ be a function from $\mathbb{N}$ into ExFormulasOf $S$. Suppose that $D$ is isotone and R1 $S, \mathrm{R} 8 S, \mathrm{R} 2 S, \mathrm{R} 5 S \in$ $D$ and LettersOf $S \backslash \operatorname{SymbolsOf}\left(X \cap\left((\text { AllSymbolsOf } S)^{*} \backslash\{\emptyset\}\right)\right)$ is infinite and $X \operatorname{addw}\left(D, n_{1}\right) \subseteq Z$ and $Z$ is $D$-consistent and $\operatorname{rng} n_{1}=$ ExFormulasOf $S$. Then $Z$ is $S$-witnessed. 


\section{Constructions for Countable Languages: Consistently Maximizing a Set of Formulas of a Countable Language (Lindenbaum's Lemma)}

Let us consider $X, S, D$ and let $p_{2}$ be an element of AllFormulasOf $S$. The functor $\left(D, p_{2}\right)$ AddFormulaTo $X$ is defined by:

$$
\left(D, p_{2}\right) \text { AddFormulaTo } X=\left\{\begin{aligned}
& X \cup\left\{p_{2}\right\} \\
& \text { if } \operatorname{xnot} p_{2} \text { is not }(X, D) \text {-provable, } \\
& X \cup\left\{\operatorname{xnot} p_{2}\right\}, \text { otherwise }
\end{aligned}\right.
$$

Let us consider $X, S, D$ and let $p_{2}$ be an element of AllFormulasOf $S$. Then $\left(D, p_{2}\right)$ AddFormulaTo $X$ is a subset of $X \cup$ AllFormulasOf $S$.

Let us consider $X, S, D$ and let $p_{2}$ be an element of AllFormulasOf $S$. Note that $X \backslash\left(\left(D, p_{2}\right)\right.$ AddFormulaTo $\left.X\right)$ is empty.

Let us consider $X, S, D$ and let $n_{1}$ be a function from $\mathbb{N}$ into AllFormulas $S$. The functor $\left(D, n_{1}\right)$ AddFormulasTo $X$ yields a function from $\mathbb{N}$ into

$2^{X \cup A l l F o r m u l a s O f} S$ and is defined by:

(Def. 74$) \quad\left(\left(D, n_{1}\right)\right.$ AddFormulasTo $\left.X\right)(0)=X$ and for every $m$ holds

$\left(\left(D, n_{1}\right)\right.$ AddFormulasTo $\left.X\right)(m+1)=$

$\left(D, n_{1}(m)\right)$ AddFormulaTo $\left(\left(D, n_{1}\right)\right.$ AddFormulasTo $\left.X\right)(m)$.

Let us consider $X, S, D$ and let $n_{1}$ be a function from $\mathbb{N}$ into AllFormulas Of $S$. The functor $\left(D, n_{1}\right)$ CompletionOf $X$ yields a subset of $X \cup$ AllFormulas Of $S$ and is defined as follows:

(Def. 75$) \quad\left(D, n_{1}\right)$ CompletionOf $X=\bigcup \operatorname{rng}\left(\left(D, n_{1}\right)\right.$ AddFormulasTo $\left.X\right)$.

Let us consider $X, S, D$ and let $n_{1}$ be a function from $\mathbb{N}$ into AllFormulasOf $S$. One can check that $X \backslash\left(\left(D, n_{1}\right)\right.$ CompletionOf $\left.X\right)$ is empty.

We now state the proposition

(19) For every relation $R$ between $2^{S \text {-sequents }}$ and $S$-sequents holds $y \in$ (FuncRule $R)(X)$ iff $y \in S$-sequents and $\langle X, y\rangle \in R$.

In the sequel $D_{2}$ is a 2-ranked rule set of $S$.

Let us consider $S$ and let $r_{1}, r_{2}$ be isotone rules of $S$. Note that $\left\{r_{1}, r_{2}\right\}$ is isotone.

Let us consider $S$ and let $r_{1}, r_{2}, r_{3}, r_{4}$ be isotone rules of $S$. Observe that $\left\{r_{1}, r_{2}, r_{3}, r_{4}\right\}$ is isotone.

Let us consider $S$. Observe that $S$-rules is isotone.

Let us consider $S$. Observe that there exists an isotone rule set of $S$ which is correct.

Let us consider $S$. Observe that there exists a correct isotone rule set of $S$ which is 2-ranked.

Let $S$ be a countable language. Observe that AllFormulasOf $S$ is countable.

We now state the proposition 
(20) Let $S$ be a countable language and $D$ be a rule set of $S$. Suppose $D$ is 2-ranked, isotone, and correct and $Z$ is $D$-consistent and $Z \subseteq$ AllFormulasOf $S$. Then there exists a non empty set $U$ and there exists an element $I$ of $U$-InterpretersOf $S$ such that $Z$ is $I$-satisfied.

In the sequel $C$ denotes a countable language and $p_{2}$ denotes a w.f.f. string of $C$.

We now state the proposition

(21) If $X \subseteq$ AllFormulasOf $C$ and $p_{2}$ is $X$-implied, then $p_{2}$ is $X$-provable.

\section{REFERENCES}

[1] Grzegorz Bancerek. Cardinal numbers. Formalized Mathematics, 1(2):377-382, 1990.

[2] Grzegorz Bancerek. The fundamental properties of natural numbers. Formalized Mathematics, 1(1):41-46, 1990.

[3] Grzegorz Bancerek. König's theorem. Formalized Mathematics, 1(3):589-593, 1990.

[4] Grzegorz Bancerek and Krzysztof Hryniewiecki. Segments of natural numbers and finite sequences. Formalized Mathematics, 1(1):107-114, 1990.

[5] Grzegorz Bancerek and Andrzej Trybulec. Miscellaneous facts about functions. Formalized Mathematics, 5(4):485-492, 1996.

[6] Czesław Byliński. Finite sequences and tuples of elements of a non-empty sets. Formalized Mathematics, 1(3):529-536, 1990.

[7] Czesław Byliński. Functions and their basic properties. Formalized Mathematics, 1(1):5565, 1990.

[8] Czesław Byliński. Functions from a set to a set. Formalized Mathematics, 1(1):153-164, 1990.

[9] Czesław Byliński. Partial functions. Formalized Mathematics, 1(2):357-367, 1990.

[10] Czesław Byliński. Some basic properties of sets. Formalized Mathematics, 1(1):47-53, 1990.

[11] Marco B. Caminati. Preliminaries to classical first order model theory. Formalized Mathematics, 19(3):155-167, 2011, doi: 10.2478/v10037-011-0025-2.

[12] Marco B. Caminati. Definition of first order language with arbitrary alphabet. Syntax of terms, atomic formulas and their subterms. Formalized Mathematics, 19(3):169-178, 2011, doi: 10.2478/v10037-011-0026-1.

[13] Marco B. Caminati. First order languages: Further syntax and semantics. Formalized Mathematics, 19(3):179-192, 2011, doi: 10.2478/v10037-011-0027-0.

[14] Marco B. Caminati. Free interpretation, quotient interpretation and substitution of a letter with a term for first order languages. Formalized Mathematics, 19(3):193-203, 2011, doi: 10.2478/v10037-011-0028-z.

[15] M.B. Caminati. Yet another proof of Goedel's completeness theorem for first-order classical logic. Arxiv preprint arXiv:0910.2059, 2009.

[16] Patricia L. Carlson and Grzegorz Bancerek. Context-free grammar - part I. Formalized Mathematics, 2(5):683-687, 1991.

[17] Agata Darmochwał. Finite sets. Formalized Mathematics, 1(1):165-167, 1990.

[18] H.D. Ebbinghaus, J. Flum, and W. Thomas. Mathematical logic. Springer, 1994.

[19] Rafał Kwiatek and Grzegorz Zwara. The divisibility of integers and integer relative primes. Formalized Mathematics, 1(5):829-832, 1990.

[20] Beata Padlewska. Families of sets. Formalized Mathematics, 1(1):147-152, 1990.

[21] Marta Pruszyńska and Marek Dudzicz. On the isomorphism between finite chains. Formalized Mathematics, 9(2):429-430, 2001.

[22] Konrad Raczkowski and Paweł Sadowski. Equivalence relations and classes of abstraction. Formalized Mathematics, 1(3):441-444, 1990.

[23] Andrzej Trybulec. Domains and their Cartesian products. Formalized Mathematics, 1(1):115-122, 1990.

[24] Andrzej Trybulec. Tuples, projections and Cartesian products. Formalized Mathematics, 1(1):97-105, 1990. 
[25] Zinaida Trybulec. Properties of subsets. Formalized Mathematics, 1(1):67-71, 1990.

[26] Edmund Woronowicz. Relations and their basic properties. Formalized Mathematics, 1(1):73-83, 1990.

[27] Edmund Woronowicz. Relations defined on sets. Formalized Mathematics, 1(1):181-186, 1990.

[28] Edmund Woronowicz and Anna Zalewska. Properties of binary relations. Formalized Mathematics, 1(1):85-89, 1990.

Received December 29, 2010 